\title{
Livedo reticularis and cerebro-vascular disease
}

\author{
W. P. STEPHENS* \\ M.B., M.R.C.P.
}

\author{
I. T. FERGUSON $\dagger$ \\ M.B., M.R.C.P.
}

\section{Departments of *Medicine and $\uparrow$ Neurology, Manchester Royal Infirmary, Manchester M13 $9 W L$}

\begin{abstract}
Summary
Three cases are described in which extensive livedo reticularis was associated with premature cerebrovascular disease. The patients presented with transient cerebral ischaemic attacks and gradually developed permanent neurological damage with intellectual impairment. The value of detailed neuropsychological testing to identify bilateral cortical abnormalities is demonstrated. CAT scanning may reveal multifocal cerebral infarction. The nature of this condition is not understood.
\end{abstract}

\section{Introduction}

Advances in non-invasive neurological diagnostic techniques have enabled the authors to study 3 comparatively young patients in whom a particularly severe cutaneous vascular disturbance was associated with neurological abnormalities.

\section{Case 1}

A 29-year-old right-handed road patrol man developed extensive livedo reticularis which was complicated by a chronic painful ulcer on the plantar aspect of the right great toe 6 years later. He was constitutionally obese and mildly hypertensive. At the age of 35 years he suddenly developed expressive dysphasia and a right facial weakness which recovered completely within 2 weeks. One year later he experienced a left hemiparesis and hemianaesthesia which partly resolved leaving residual difficulty with intricate finger movements. He had noticed memory impairment and problems of sight in his right visual field.

Following a minor road traffic accident without head injury (age 37 years) he was referred to hospital where neurological examination revealed a right homonymous hemianopia and left-sided pyramidal signs including mild weakness (MRC Grade 4) hyperreflexia and an extensor plantar response. Florid livedo reticularis covered his trunk and limbs.

Detailed haematological, biochemical and serological investigations were normal. Computerized axial tomography (CAT) showed low density areas in both parieto-occipital areas which did not: enhance following contrast administration con- 3 sistent with multiple small cerebral infarctions (Fig. 1).

Over the next 6 months he experienced several $\sigma$ sudden dysphasic episodes, lasting up to $10 \mathrm{~min}-$ during which time he reported an improvement in the hemianopia. He complained of progressive difficulty in dressing and often lost his way both outside and within the confines of his own home. $\mathbb{O}$ At the age of 38 years he experienced a deep nagging ache in both thighs with wasting of both quadriceps and Grade 4 weakness of left hip flexion (MR6, 1976). Knee jerks were absent in contrast to alष other tendon reflexes which were brisk. Boł? plantar responses were now extensor, indicatiog additional recent right-sided pyramidal tract affection. There was no sensory loss. The visual field $\bar{\partial}$ defect was now a right inferior homonymous quadrantanopia. A second CAT scan was un- $\stackrel{\unrhd}{\varrho}$ changed.

Intellectual assessment revealed a marked dis- $\frac{0}{3}$ crepancy between verbal (114) and performance (66) i.q. on the Wechsler Adult Intelligence Scale (WAIS) suggesting non-dominant hemisphere dysfunction. He showed major visuo-spatial and visual 3 . retention problems but despite prior transient dysphasic episodes there was no linguistic abnormality detected on specific testing.

A skin biopsy showed a mild perivascular chronic inflammatory infiltrate with no evidence of vasculitis. 을 A muscle biopsy taken from the right quadriceps showed normal muscle and a normal medium sizedo artery. Concentric needle electromyography of the $\frac{\bar{N}}{\mathrm{~N}}$ left quadriceps muscle showed a typical denervation o pattern with fibrillation, positive sharp waves and $N$ reduced interference pattern. Other muscle samples $\mathbb{C}_{\mathrm{J}}$ and nerve conduction velocities were within the normal range. An elestrocardiogram showed first degree heart block (PR interval 0.36 sec) which had not been present one year before. An echocardio- $\stackrel{\infty}{\rightarrow}$ gram revealed poor movement of the posterior left ventricular wall unassociated with valvular disease. 

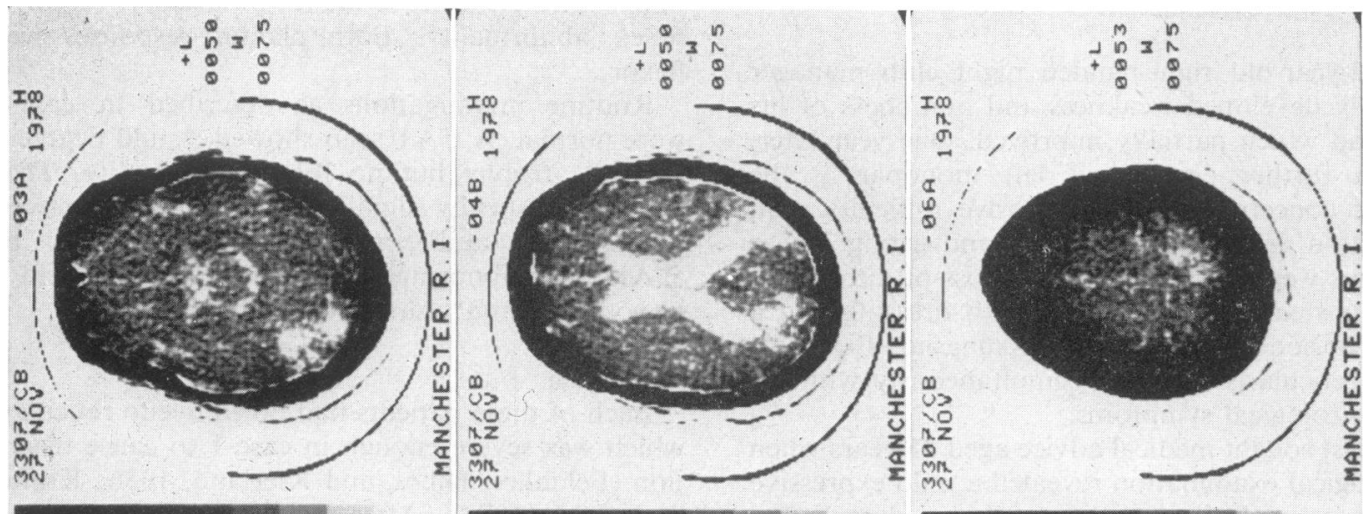

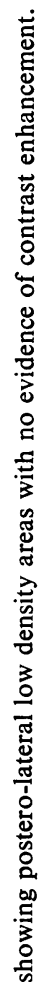
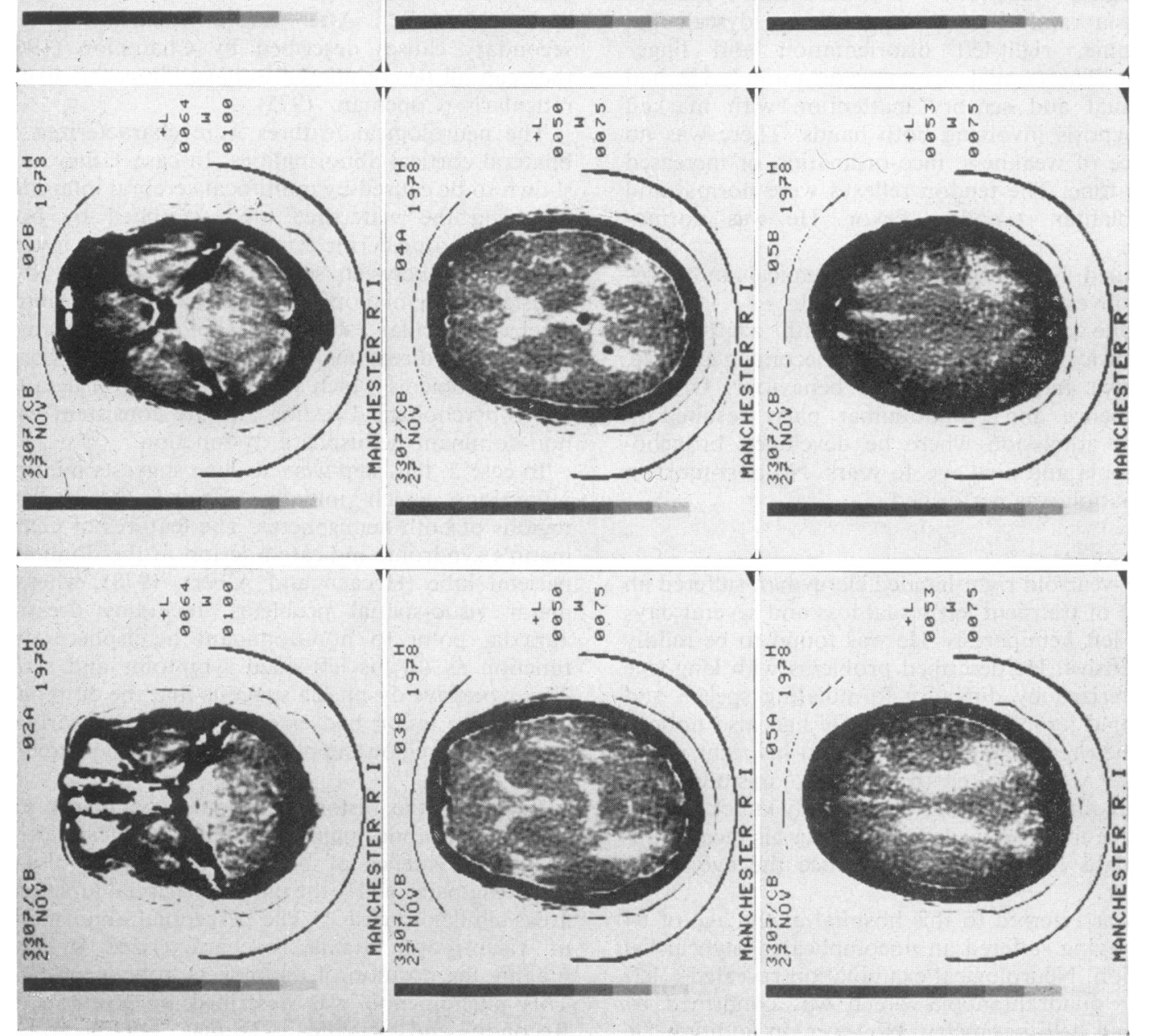

4

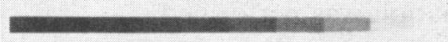


The ejection fraction was at the lower limit of normal.

\section{Case 2}

A 42-year-old right-handed night club manager suddenly developed wéakness and numbness of his left hand which partially improved. One year later, after a further episode of left monoparesis, he became concerned over progressive difficulty with calculation and deterioration in handwriting. These problems were compounded by a loss of directional sense, unsteady gait, difficulty with dressing and a single episode of transient dysphasia. Extensive livedo reticularis appeared simultaneously with his first neurological symptoms.

He first sought medical advice aged 45 years when neurological examination revealed a mild expressive dysphasia and dysarthria, striking dyscalculia, dysgraphia, right-left disorientation and finger agnosia. The cranial nerves were normal. He had left visual and sensory inattention with marked astereognosis involving both hands. There was no evidence of weakness, inco-ordination or increased muscle tone. The tendon reflexes were normal and both plantar responses flexor. He was normotensive.

Detailed haematological, biochemical and serological investigations were unhelpful.

Despite empirical treatment with azathioprine $50 \mathrm{mg}$ thrice/day, he deteriorated, becoming irritable, aggressive and childish in his behaviour. Urinary incontinence and pseudobulbar palsy resulted in hospital admission where he developed bronchopneumonia and died age 46 years. No post-mortem examination was performed.

\section{Case 3}

A 63-year-old right-handed clergyman suffered an episode of transient left visual loss and several days later a left hemiparesis. He was found to be mildly hypertensive. He described problems with language characterized by difficulty formulating speech and occasional jumbling of words. He had also noticed that when reaching for an object in his right visual field he would often be clumsy, misjudge the position of the object and occasionally knock it over. Extensive livedo reticularis involving his face, trunk and thighs had been present since the age of 58 years.

He was referred to this hospital at the age of 64 years having suffered an uncomplicated myocardial infarction. Neurological examination revealed a left inferior quadrantanopia which was confirmed by Goldman field perimetry. However, in addition, he was unable to locate the position of objects in his right visual field, either with his right or left hand whilst maintaining central fixation (so-called visuo- motor ataxia). Pursuit and voluntary eye movements were full. The remainder of the cranial nerves were $\stackrel{\mathbb{Q}}{\complement}$ intact. There was no evidence of sensory, motor or $C$ reflex abnormality. Both plantar responses were $\vec{F}$ flexor.

Routine investigations as described in case 1 음 were normal. A CAT scan showed a mild degree of $\frac{\bar{\sigma}}{\bar{\omega}}$ cortical atrophy but no focal abnormality. There $\frac{\rho}{\Phi}$ was a statistically significant discrepancy between $\varnothing$ verbal (127) and performance (99) i.q. on the WAIS. The abnormal features were in the field of $\overrightarrow{0}$ visuospatial and visual memory function.

\section{Discussion}

Each of these patients had florid livedo reticularis which was severe enough in case 1 to cause ulceration (Feldaker, Hines, and Kierland, 1956; Klüken 0 and Gerl, 1972). After careful exclusion of the 9 secondary causes described by Champion (1965) के they can all be considered to have idiopathic livedo $\partial$ reticularis (Copeman, 1975).

The neurological features were characterized by bilateral cortical abnormalities. In case 1 they were T shown to be caused by multifocal cerebral infarction involving the watershed areas supplied by both anterior and posterior cerebral arteries. The inverse $₹$ relationship between several of the transient dy\& $\vec{\varphi}$ phasic and hemianopic attacks may indicate intraํ cerebral vascular 'stealing'. The dressing apraxi and loss of directional sense, both point to visuow spatial problems which were confirmed on detailed neuro-psychological testing and are consistent with non-dominant hemisphere dysfunction.

In case 2 , the 'step-wise' decline suggests multiple infarctions which initially affected the parietal $\overrightarrow{\overrightarrow{0}}$ regions of both hemispheres. The features of Gerstmann's syndrome indicate a lesion in the dominant parietal lobe (Hécaen and Albert, 1978), whereas major visuo-spatial problems, including dressing apraxia, point to non-dominant hemisphere dysfunction as do his left-sided symptoms and signs. The expressive dysphasia suggests that the dominant hemisphere lesion had extended more anteriorly to include the region supplied by the middle cerebral artery.

In case 3 the history of linguistic difficulty and $D$ visuospatial abnormalities on psychological testing provided evidence of bilateral hemisphere disease $N$ involving particularly the parieto-occipital junctional areas similar to case 1 . The perceptual abnormality 0 of visuo-motor ataxia is characterized by mis- $\underset{\omega}{ }$ judging the position of the objects in his right field. This phenomenon was described by Rondot, De 0 Recondo and Ribadeau Dumas (1977) and is $\Phi$ associated with bilateral parieto-occipital disease. $\stackrel{\odot}{+}$

Case 1 was further complicated by a clinical 0 picture suggesting a femoral nerve infarction 
(Asbury, 1977) and a cardiac abnormality consistent with cardiomyopathy due to a generalized small blood vessel disorder (Oakley, 1972).

A detailed search of the world literature reveals sporadic reports of very similar cases which previously have never been collected together (Barker, Hines and Craig, 1941; Champion and Rook, 1960; Church, 1962; Sneddon, 1965; Strzałko, Rymczonek and Zajgner, 1966; Trzebicki and Buksowicz, 1969; Buksowicz, Niedzielska and Trzebicki, 1970; Gralewski, 1972; Piñol Aguadé et al., 1975; Pellat et al., 1976; Rumpl and Rumpl, 1979). The mean age of onset of the first neurological symptom in these 21 patients (including the present 3 ) was 35 years with a range of 16 to 63 years. Livedo reticularis preceded the first neurological incident in $55 \%$ and in the remainder it appeared simultaneously or shortly afterwards. The patient was usually obese and $60 \%$ had mild hypertension. There was a male to female preponderance of $1.6: 1$.

The clinical presentation was of recurrent transient ischaemic attacks in a young to middle-aged person causing hemipareses, hemianaesthesia, visual field defects and language disorders. Uncommon presentations included focal epilepsy and transient global amnesia. A more chronic form occurs characterized by diffuse cognitive impairment which may be confirmed by an appropriate test such as the WAIS. Five out of the 21 cases died of their neurological disease within 9 years. In the remainder, there was a tendency for permanent neurological deficit to supervene as the length of follow-up increased.

The limited amount of pathological data available (Pellat et al., 1976) casts little light on the pathophysiology of this clinical association and detailed post-mortem studies are required. However, these will not be forthcoming unless the occurrence of this condition is more widely acknowledged. Speculations on the aetiology have included cerebral thromboangiitis obliterans (Miller-Fisher, 1957) and a 'vasculopathy' of obscure aetiology (Sneddon, 1965). Arteritis has usually been fully excluded (Fauci, Haynes and Katz, 1978).

If a young to middle-aged person presenting with transient ischaemic attacks is found to have widespread livedo reticularis, then evidence of unsuspected diffuse abnormalities should be sought by detailed neuropsychological testing (McFie, 1979).

\section{Acknowledgments}

We are grateful to Professor S. W. Stanbury for permission to present cases 1 and 3 , and to Dr D. Gaan for permission to present case 2 . We would like to thank Drs R. G. Las- celles and W. J. K. Cumming for help in the preparation of this paper and Professor I. Isherwood for the CAT scan.

\section{References}

Asbury, A.K. (1977) Proximal diabetic neuropathy. Annals of Neurology, 2, 179.

Barker, N.W., Hines, E.A. \& Craig, W. (1941) Livedo reticularis: a peripheral arteriolar disease. American Heart Journal, 21, 592.

Buksowicz, C., Niedzielska, K. \& Trzebicki, J. (1970) [The syndrome of livedo racemosa with cerebral signs.] Neurologia i neurochirurgia. Poland, 4, 587.

Champion, R.H. (1965) Livedo reticularis-a review. British Journal of Dermatology, 77, 167.

Champion, R.H. \& Rook, A. (1960) Livedo reticularis. Proceedings of the Royal Society of Medicine, 53, 961.

Church, R.E. (1962) Reticular livedo with cerebrovascular lesions. British Journal of Dermatology, 74, 156.

Copeman, P. W. M. (1975) Livedo reticularis. British Journal of Dermatology, 93, 519.

Fauci, A.S., Haynes, B.F. \& Katz, P. (1978) The spectrum of vasculitis. Annals of Internal Medicine, 89, 660.

Feldaker, M., Hines, E.A. \& Kierland, R.R. (1956) Livedo reticularis with ulceration. Circulation, 13, 196. GRALEWSKI, Z. (1972) [Livedo racemosa and organic cerebral changes.] Neurologia, neurochirurgia i psychiatria polska, 22, 293.

Hécaen, H. \& Albert, M.L. (1978) In: Human Neuropsychology, p. 310 . J. Wiley \& Sons, New York.

KLÜKEN, N. \& GeRL, R. (1972) [Aetiopathogenic and clinical observations in livedo racemosa.] Zeitschrift fur Haut und Geschlechtskrankheit, 47, 807.

McFIE, J. (1979) In: Progress in Stroke Research I. (Ed by Greenhalgh, R.M. \& Clifford Rose, F.) pp. 144-149. Pitman Medical Press, Bath.

Medical Research Council (1976) Memorandum no. 45, H.M. Stationery Office, London.

Miller-FISHer, C. (1957) Cerebral thromboangiitis obliterans. Medicine. Baltimore, 46, 169.

OAKLEY, C.M. (1972) Clinical definitions and classification of cardiomyopathies, Postgraduate Medical Journal, 48, 703.

Pellat, J., Perret, J., Pasquier, B., Crouzet, G., Dubos, G. \& CHATEAU, R. (1976) [Anatomical and angiographic study of a case of disseminated thrombo-angiitis with predominant cerebral manifestations.] Revue Neurologique. Paris, 132, 517.

Piñol Aguade, J., Ferrandiz, C., Ferrer Roca, O. \& INGELMO, M. (1975) [Livedo reticularis and cerebrovascular accidents.] Medicina Cutanea Ibero-LatinoAmericana. Lisbon, 3, 257.

Rondot, P., De Recondo, J. \& Ribadeau Dumas, J.L. (1977) Visuomotor ataxia. Brain, 100, 355.

Rumpl, E. \& RuMPL, H. (1979) Recurrent transient global amnesia in a case with cerebrovascular lesions and livedo reticularis. Journal of Neurology, 221, 127.

SNEDDON, I.B. (1965) Cerebro-vascular lesions and livedo reticularis. British Journal of Dermatology, 77, 180,

StrZAŁKo, M., RYMCZoneK, C. \& ZAJGNeR, J. (1966) [Angiographic examinations in a case of livedo racemosa and organic changes in the brain.] Neurologia, neurochirurgia $i$ psychiatria polska, 16, 363.

TRZeBICKI, J. \& Buksowicz, C. (1969) [Cerebral angiography in livedo racemosa.] Fortschritte auf dem Gebiete der Röntgenstrahlen und der Nuklearmedizin, 111, 704. 